\title{
Effects of sport expertise on representational momentum during timing control
}

\author{
Hiroki Nakamoto • Shiro Mori • Sachi Ikudome • \\ Satoshi Unenaka $\cdot$ Kuniyasu Imanaka
}

Published online: 25 December 2014

(C) The Psychonomic Society, Inc. 2014

\begin{abstract}
Sports involving fast visual perception require players to compensate for delays in neural processing of visual information. Memory for the final position of a moving object is distorted forward along its path of motion (i.e., "representational momentum," RM). This cognitive extrapolation of visual perception might compensate for the neural delay in interacting appropriately with a moving object. The present study examined whether experienced batters cognitively extrapolate the location of a fast-moving object and whether this extrapolation is associated with coincident timing control. Nine expert and nine novice baseball players performed a prediction motion task in which a target moved from one end of a straight $400-\mathrm{cm}$ track at a constant velocity. In half of the trials, vision was suddenly occluded when the target reached the $200-\mathrm{cm}$ point (occlusion condition). Participants had to press a button concurrently with the target arrival at the end of the track and verbally report their subjective assessment of the first target-occluded position. Experts showed larger RM magnitude (cognitive extrapolation) than did novices in the occlusion condition. RM magnitude and timing errors were strongly correlated in the fast velocity condition in both experts and novices, whereas in the slow velocity condition, a
\end{abstract}

H. Nakamoto $(\square) \cdot$ S. Mori $\cdot$ S. Ikudome

Faculty of Physical Education, National Institute of Fitness and Sports in Kanoya, 1 Shiromizu, Kanoya, Kagoshima 891-2393, Japan

e-mail: nakamoto@nifs-k.ac.jp

S. Unenaka

Graduate School of Physical Education, Doctor's Course, National Institute of Fitness and Sports in Kanoya, 1 Shiromizu, Kanoya, Kagoshima 891-2393, Japan

K. Imanaka

Department of Kinesiology, Graduate School of Science,

Tokyo Metropolitan University, 1-1 Minamiosawa, Hachioji,

Tokyo 192-0397, Japan significant correlation appeared only in experts. This suggests that experts can cognitively extrapolate the location of a moving object according to their anticipation and, as a result, potentially circumvent neural processing delays. This process might be used to control response timing when interacting with moving objects.

Keywords Representational momentum · Sport expertise · Visuo-motor control

\section{Introduction}

How a moving object is processed, and how the processed information is used for motor control of intercepting (e.g., catching, hitting) the moving object, is a major concern in understanding the human visuo-motor system. The visual system has some limitations, particularly concerning good performance in sports requiring fast visual perception (e.g., baseball and tennis).

One limitation of the visual system is neural delay, that is, the time interval between stimulation and resultant change in neural activity at the relevant brain site (Nijhawan, 2008; Zago, McIntyre, Senot, \& Lacquaniti, 2009). The initial stages of visual processing may necessarily incur a delay greater than tens of milliseconds, which may induce an error between the physical position of an object and its perceived position: more than $4 \mathrm{ft}(1.2 \mathrm{~m})$ for a tennis ball travelling at $60 \mathrm{mph}$ (96.6 kph), and $13.2 \mathrm{ft}(4.02 \mathrm{~m})$ for a cricket ball traveling at $90 \mathrm{mph}$ (144.84 kph) (Nijhawan, 2008; Nijhawan \& Wu, 2009). Another primary limitation relates to our visual hardware. For instance, Hubbard and Seng (1954) reported that the line of gaze for professional baseball batters is decoupled from the ball $240 \mathrm{~ms}$ prior to bat-ball contact. This is because the angular velocity of the pitched ball exceeds the limit of angular velocity of pursuit eye movement (see also in other sports, 
Croft, Button, \& Dicks, 2010; Lee, 2010; Ripoll \& Fleurance, 1988; Singer, Williams, Frehlich, Janelle, Radlo, Barba, \& Bouchard, 1998). A large anticipatory saccade is thus needed to shift gaze direction along the anticipated trajectory of a pitched ball until the point of bat-ball contact (Bahill \& LaRitz, 1984; Land \& McLeod, 2000; Ripoll \& Fleurance, 1988; Singer et al., 1998). During saccadic eye movement, visual information is suppressed and/or degraded (Campbell \& Wurtz, 1978; Volkmann, Schick, \& Riggs, 1968).

Therefore, at least physically, no batter is able to obtain the correct information of the real ball position due to neural processing delays and limits to visual hardware. Nonetheless, timing control in the interception of a fast-moving object is often accurate and does not lag behind the real-world object position. Thus, it is likely that expert athletes compensate for delays in neural processing and fill perceptual gaps in order to interact optimally with a fast-moving target.

One possible cognitive mechanism that could overcome such limitations is cognitive extrapolation in appropriately anticipating the future location of a moving object (DeLucia \& Liddell, 1998; Hubbard, 2005; Nijhawan, 2008; Nijhawan $\& \mathrm{Wu}, 2009$; Tresilian, 1995). Memory of the final position of a moving object is displaced forward along its path of motion; this effect is referred to as representational momentum (RM; Freyd, 1983; Freyd \& Finke, 1984; Hubbard \& Bharucha, 1988). In the seminal work by Hubbard and Bharucha (1988), observers were presented with objects that continuously moved either horizontally or vertically and then suddenly vanished. After the target had vanished, the observers operated a computer mouse to position a cursor on the computer display where they perceived that the target had vanished. They found that observers were more likely to place the cursor at a location slightly further along the anticipated trajectory. Further, RM magnitude for moving objects is modulated by several visual and/or cognitive factors, such as conceptual knowledge about target identity (e.g., RM magnitude for the moving figure of a church was smaller than that for moving figure of a rocket because the typical immobility of the church overrides a general bias for RM; Reed \& Vinson, 1996), expectations regarding future target motion (Hubbard \& Bharucha, 1988; Verfaillie \& d'Ydewalle, 1991), and an object's motion velocity (Hubbard \& Bharucha, 1988; Munger $\&$ Minchew, 2002). These studies suggest that the human visual system extrapolates the future location of a moving object on the basis of both the object's physical characteristics and the observer's knowledge base. This forward displacement may be the mechanism needed to overcome the limitations of the visuo-motor system when participating in fast ball sports (Hubbard, 2005; Marinovic, Plooy, \& Arnold, 2012; Nijhawan, 2008; Nijhawan \& Wu, 2009).

Recent studies have shown that the domain-specific nature of expertise also modulates RM magnitude. It has been reported that when participants watched scenes of simulated road driving or aircraft landing scenes, experienced automobile drivers and aviation pilots had a larger forward displacement than did inexperienced individuals (Blättler, Ferrari, Didierjean, van Elslande, \& Marmèche, 2010; Blättler, Ferrari, Didierjean, \& Marmèche, 2011; Blättler, Ferrari, Didierjean, \& Marmèche, 2012). In the sports domain, Gorman, Abernethy, and Farrow (2011) reported that when expert basketball players were provided with a static schematic or a moving video from their domain of expertise, the players perceived the next likely state of the play pattern (see also, Didierjean \& Marmèche, 2005; Gorman, Abernethy, \& Farrow, 2012). These studies indicate that knowledge acquired through years of experience with a particular behavioral domain (e.g., sports) modulates RM magnitude on perceptual judgments in the domain of expertise, and that experts in a particular domain have a more refined ability to extrapolate future events than do novices. In fact, several studies assessing expertise in sports have shown that experts develop the anticipation ability that allows anticipation of a few hundred milliseconds (for reviews, see Williams \& Ericsson, 2005; Williams, Davids, \& Williams, 1999). Therefore, it is likely that experts in fast ball sports develop their cognitive extrapolation ability to anticipate the future location of a fast-moving object, thus overcoming limitations in the visual system. However, the question of whether cognitive extrapolation for fastmoving objects is modulated by expertise in fast ball sports has not yet been studied [however, see Gilis et al. (2008) for similar studies with soccer].

Although most studies using an RM paradigm for a moving target have involved perceptual judgments that require participants to represent the vanished location only of a moving target, the tasks (e.g., prediction-motion tasks; PM) have often been used to understand visuo-motor coordination for interceptive timing control. In a PM task, participants view a moving target that is subsequently occluded, and participants then respond when the moving target would arrive at a goal if the target continued moving even after it was occluded from the observer's sight. A number of studies using the PM task have revealed that experts have superior timing ability compared with novices (Akpinar, Devrilmez, \& Kirazci, 2012; Ripoll \& Latiri, 1997; Tenenbaum, Sar-El, \& Bar-Eli, 2000) due to accurate estimation of the time-to-contact (TTC), which is one of the critical factors for correctly intercepting a moving object (Tresilian, 1995). DeLucia and Liddell (1998) reported that cognitive motion extrapolation for the future location of a moving target after it disappears is associated positively with TTC estimation. That is, superior timing control among expert athletes during a PM task may be attributable to appropriate cognitive extrapolation of the moving target.

Gray and Thornton (2001) reported that TTC underestimation (i.e., early response), which often occurs in PM tasks, did not occur when the RM was absent by using a display that disambiguated the location of target disappearance. In other 
words, there is a link between RM (i.e., localization errors) and TTC, although their study showed that cognitive motion extrapolation of a future target position induces larger temporal error during a PM task. However, Gray and Thornton's study was conducted with a longer TTC duration $(>1 \mathrm{~s})$ that did not require the fast perception necessary in, for instance, baseball batting $(<0.4 \mathrm{~s})$. TTC underestimation is often greater within a longer TTC (i.e., a slow target) relative to a shorter TTC condition (i.e., a fast target) (Schiff \& Detwiler, 1979; Schiff \& Oldak, 1990). Moreover, RM magnitude increases with increasing target speed (Freyd \& Finke, 1985). If cognitive extrapolation is the mechanism compensating for neural delays in fast visual perception, it becomes more useful in interacting with a faster-moving object. Thus, if cognitive extrapolation develops with sport experience to avoid neural delays, there would be a stronger positive link for TTC estimation accuracy of a fast-moving object than of a slowmoving object.

Thus, the purpose of the present study was to determine whether experience with fast ball sports helps to develop cognitive extrapolation ability, and whether this ability is related to superior coincidental timing performance, especially when facing a fast-moving object. To this end, a buttonpress PM task was used, as performance would be determined entirely by the ability to accurately estimate the TTC of the moving target that might be determined by RM and response onset timing (Tresilian, 1995). If experience with fast ball sports develops cognitive extrapolation ability, expert fast ball sport athletes who excel at the PM task should show larger RM magnitude compared with novices. Moreover, if expert athletes compensate for delays in neural processing and fill the perceptual gap with cognitive extrapolation, the extrapolated information should be used to interact optimally with a moving target. That is, we predicted that there would be a strong correlation between RM magnitude and PM task performance. Moreover, the correlation will be more prominent in response to fast-moving objects compared with relatively slow moving objects.

\section{Methods}

\section{Participants}

Eighteen male college students aged 20-22 years participated in this experiment. All participants had normal or correctedto-normal vision. Nine students belonged to the official college baseball team (more than 10 years playing experience). Participants generally spent $20 \mathrm{~h}$ per week practicing or playing actual games. The other nine participants were college students who played baseball but had not received any special baseball training. Participants consented to take part in this experiment after being informed of the experimental procedures; however, participants were not informed about the goals of the experiment. The ethics committee of the National Institute of Fitness and Sports in Kanoya approved this study; the experiment was conducted in accordance with the principles set forth in the Declaration of Helsinki.

\section{Experimental apparatus}

The experimental device used for the RM/PM task was a horizontal electronic trackway (400 cm in length, located $90 \mathrm{~cm}$ above the ground), with 200 light-emitting diodes (LEDs: $\varphi 5 \mathrm{~mm}$ in size) that simulated the linear motion of objects (Fig. 1) (AO-5N model; Applied Office, Tokyo, Japan). The LEDs, spaced at 2-cm intervals, were turned on and off rapidly in sequence (i.e., ISI $=0$ ), and participants were able to perceive the continuous motion of an approaching target. The target velocity was modified by instantaneously changing the LED on and off times (i.e., each LED was illuminated for one two-hundredth of the total time of target motion). In addition, a manual switch (10 cm high, $3 \mathrm{~cm}$ wide, and $2 \mathrm{~cm}$ deep) that fit in the palm of an adult hand was used to register participants' coincident timing response (i.e., when the light reached the end of the track, participants were asked to push the button with their thumb).

\section{Experimental task and procedure}

The moving target moved from one end of the trackway at a constant velocity (slow: $10 \mathrm{~m} / \mathrm{s}$ or fast: $15 \mathrm{~m} / \mathrm{s}$ ) after the presentation of a warning stimulus for $3 \mathrm{~s}$. In half of the trials, the target was not occluded (non-occlusion condition), and in the other half, the target was suddenly occluded halfway down the trackway (i.e., $200 \mathrm{~cm}$ ), irrespective of stimulus velocity (occlusion condition). The viewing time periods for the moving target in each occluded condition are summarized in Table 1. Previous studies show that after a pitch is delivered in baseball, the ball reaches the batter in around 400-800 ms (Frohlich, 1984; Selin, 1959; Williams \& Underwood, 1986). Therefore, the total time of the target presentation was devised in such a way as to create a time scale similar to that which a baseball batter generally faces in actual batting (i.e., slow condition). In this case, however, the actual velocity in the batting task was not replicated in the experiment task because the actual target (i.e., baseball) traveling distance is about $16 \mathrm{~m}$, whereas the distance in the present task was $4 \mathrm{~m}$. Therefore, in order to create a relatively faster condition within the visual reaction time, the degree of velocity difference between the slow $(10 \mathrm{~m} / \mathrm{s})$ and fast $(15 \mathrm{~m} / \mathrm{s})$ conditions was set at $50 \%$, well above the threshold value that is generally found to be sufficient to allow the perception of a velocity difference (Benguigui, Ripoll, \& Broderick, 2003; Le Runigo, Benguigui, \& Bardy, 2005). 


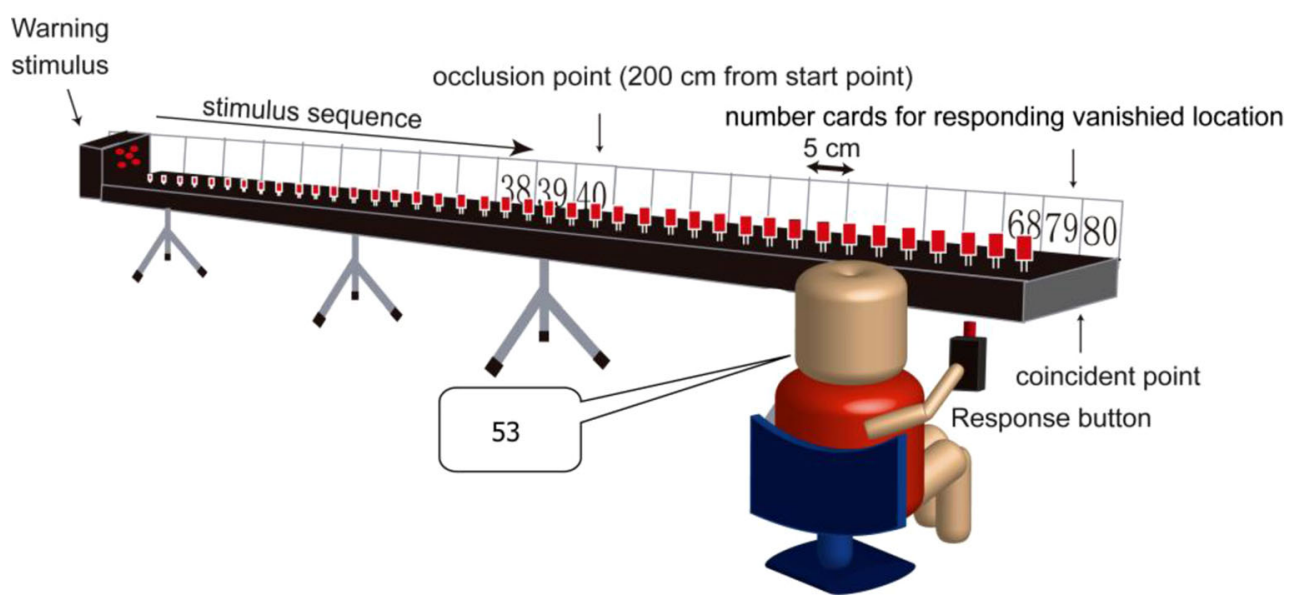

Fig. 1 The position of the study participant in relation to the electronic trackway during displacement of the visual target. Participants manually pressed a button coinciding with the arrival of an apparent moving target at the end of trackway, which ran on a straight trackway. After the button press, participants verbally reported where the moving target vanished by indicating the card number placed beside the electronic trackway
In both the non-occlusion and occlusion condition, the primary experimental task involved a PM task that required a manual button press coinciding with the arrival of an apparent moving target at the end of the trackway (in the occlusion condition, although the target disappeared at the halfway point of the trackway, participants responded with a manual button press when they felt that the moving target would have arrived at the end of the trackway after it disappeared). The secondary task involved judgment of subjective vanishing location (i.e., $\mathrm{RM}$ task) after the coincident timing response (in the nonocclusion condition, although the target always continued until the end of the trackway, participants had to indicate the last location on the trackway (if this was their perception) in order to standardize the method of response between the conditions).

Participants were seated comfortably to the right side of the edge of the trackway at a distance of $100 \mathrm{~cm}$ and held a pushbutton in their dominant hand (Fig. 1). Participants were familiarized with the experimental procedure before providing written informed consent. Next, participants practiced four stimulus conditions (occluded and non-occluded conditions in each velocity condition). Each condition comprised 10 trials (40 practice trials in total) in a random order.

Participants were provided 80 experimental trials in a random combination of each stimulus condition; 40 trials were performed in the occlusion and 40 in the non-occlusion condition (there was no difference between the occluded trials and

Table 1 Summary of visible and invisible time in the slow and fast conditions with target occlusion

\begin{tabular}{llll}
\hline Velocity condition & Visible time & Invisible time & Total time \\
\hline Slow condition & $200 \mathrm{~ms}$ & $200 \mathrm{~ms}$ & $400 \mathrm{~ms}$ \\
Fast condition & $133 \mathrm{~ms}$ & $133 \mathrm{~ms}$ & $266 \mathrm{~ms}$ \\
\hline
\end{tabular}

the non-occluded trials except for the occlusion). This established a $50 \%$ chance of target occlusion, which prevented confounding the effects of occlusion with differences in a priori probability or subjective expectancy predicted by the participants. In order to estimate subjective vanishing location, participants verbally reported where the moving target vanished with a card number placed beside the electrical trackway (Fig. 1) after the coincident timing response with a button press (the card number of the physically vanishing location in occlusion trials was 40 ). The cards were located at $5-\mathrm{cm}$ intervals from the start points of the moving target. Therefore, subjective vanishing location was computed by multiplying the reported card number by $5 \mathrm{~cm}$. When participants perceived that the target had reached the end of trackway, they reported verbally the card number 80 placed beside the end of electrical trackway (i.e., $400 \mathrm{~cm}$ from the start point). In order to determine appropriate intervals for the measurement of the vanishing location, we used a variable interval starting at $1 \mathrm{~cm}$ with $1-\mathrm{cm}$ increments as a preliminary study. In this case, participants were required to recall both the subjective vanishing location and their level of confidence in their response ("confident," "neither," or "not confident"). "Not confident" and "neither" responses disappeared above the 5-cm interval. Therefore, we used a $5-\mathrm{cm}$ interval in the present experiment. Moreover, in order to eliminate differences in visual target-tracking strategies between participants, participants were required to track the moving target from the start to the end of the track during the task. Participants were not informed of the results regarding temporal errors or vanished locations for each trial.

Measurements and analysis

RM magnitudes were calculated by subtracting the actual vanishing location (i.e., $200 \mathrm{~cm}$ ) from the reported subjective 
vanishing location in the occlusion condition. Mean RM magnitudes were subjected to mixed measures (group: baseball vs. control) $\times 2$ (velocity: slow vs. fast) ANOVAs, with group as a between-subjects factor and velocity as withinsubjects factor.

Constant timing error (CTE) was defined as the duration between the target arrival signal generated by the computer controlling the trackway and the button press signal (i.e., a bias in the response timing of when the target would reach the end of the trajectory). Positive CTE values indicate a late response, and negative values indicate an early response. To calculate CTE, we measured the duration from the target arrival signal to the button press signal, which was sampled at 1,000 Hz. Mean CTEs were subjected to mixed measures (group: baseball vs. control) $\times 2$ (velocity: slow vs. fast) $\times 2$ (occlusion: occlusion vs. non-occlusion) ANOVAs with group as a between-subjects factor and velocity and occlusion as within-subjects factors. Degrees of freedom for all $F$ ratios were adjusted using the Greenhouse-Geisser procedure.

A correlation analysis for RM magnitude and CTEs in the occlusion condition was performed in order to investigate whether RM magnitude influenced coincident timing performance.

\section{Results}

Expert-novice differences in RM magnitude

Figures 2 and 3 show the probability of a specific response as a function of distance and the mean subjective vanishing location, respectively, for the baseball and control groups. The mean RM magnitude that was calculated by subtracting the actual vanishing location (i.e., $200 \mathrm{~cm}$ ) from the reported subjective vanishing location in the occlusion condition was subjected to a two-way mixed ANOVA. There were significant main effects of velocity $\left[F(1,16)=9.97, P<.01, \eta_{\mathrm{p}}{ }^{2}=\right.$ $.38]$ and group $\left[F(1,16)=21.88, P<.001, \eta_{\mathrm{p}}{ }^{2}=.58\right]$, but no

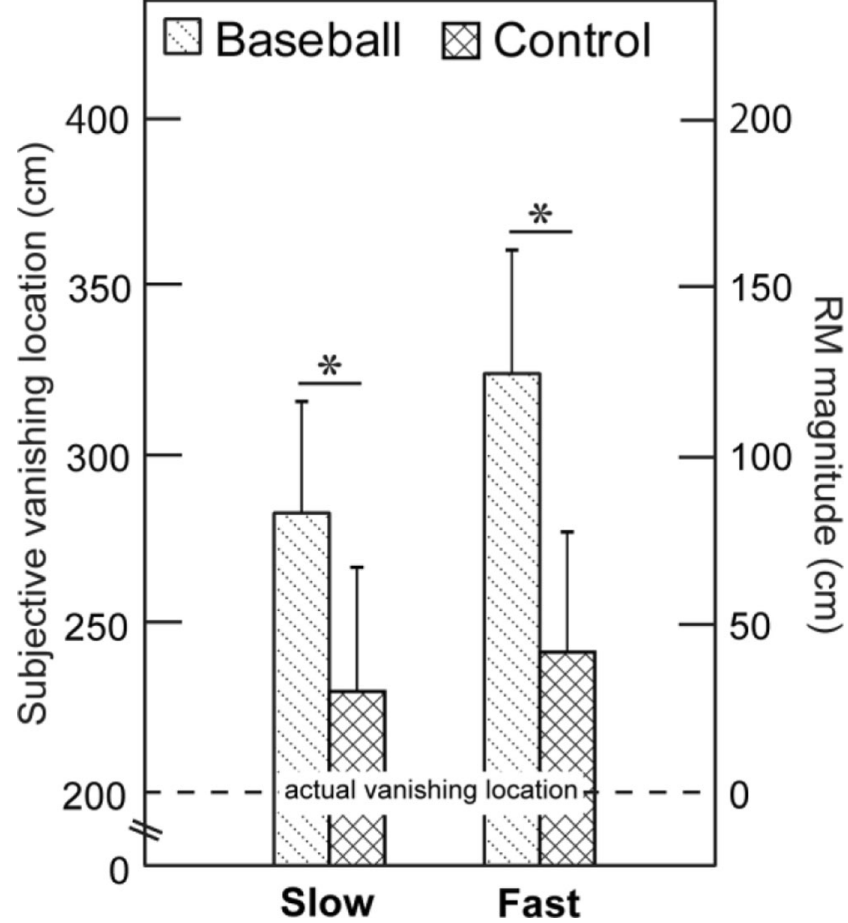

Fig. 3 Mean subjective vanishing location and representational momentum (RM) magnitude in each velocity condition. Vertical bars Standard deviation, horizontal dashed line actual vanishing location. RM magnitudes were calculated by subtracting the actual vanishing location (i.e., $200 \mathrm{~cm}$ ) from the reported subjective vanishing location in the occlusion condition

interaction $\left[F(1,16)=3.09, P=.098, \eta_{\mathrm{p}}^{2}=.16\right]$. These results indicate that expert baseball players subjectively perceived a farther target location (i.e., more target motion) after the target vanished as compared with the control group.

Next, the constant occlusion of the stimulus at $200 \mathrm{~cm}$ might lead to a large learning effect. Given that participants no doubt became aware of the fact that the stimulus would either vanish halfway along its trajectory or vanish at the end of its trajectory, participants were able to improve their performance with practice on the task. If practice effects exist in judgments of the target vanishing location, the assertion that
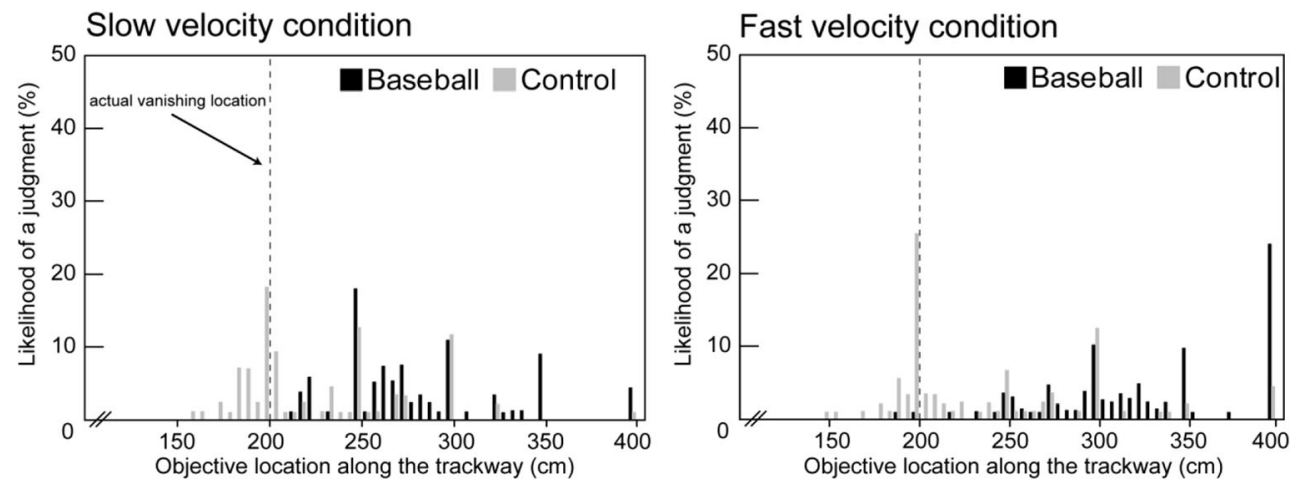

Fig. 2 Probability of response for the subjective vanishing location within the occlusion condition. Vertical dashed line Actual vanishing location 
experts in fast ball sports can extrapolate the future location of a fast-moving object might be compromised. Therefore, in order to preclude the possibility of practice effects, we analyzed RM magnitude in the occlusion condition from the initial trial to the final trial (data only in the occlusion condition were extracted from random trial sequences). RM magnitude was subjected to a mixed measures 2 (group: baseball vs. control) $\times 2$ (velocity: slow or fast) $\times 10$ (trial: first trial through final trial) ANOVA. There was no significant main effect of trial $\left[F(9,144)=1.26, P=.26, \eta_{\mathrm{p}}^{2}=.07\right]$, nor were there any significant interactions regarding the trial factor. According to these results (see also Fig. 2), practice effects did not exist in the present study with regard to RM magnitude.

\section{Relationship between RM magnitude and temporal errors}

Figure 4 shows the mean CTE of button-press responses for both groups. The mean CTE was subjected to a mixed threeway ANOVA. There was a significant main effect of velocity $\left[F(1,16)=62.67, P<.001, \eta_{\mathrm{p}}{ }^{2}=.80\right]$, as well as significant velocity $\times$ group $\left[F(1,16)=8.47, P<.01, \eta_{\mathrm{p}}^{2}=.35\right]$ and velocity $\times$ occlusion $\times$ group interactions $[F(1,16)=7.72, P<$ $\left..05, \eta_{\mathrm{p}}{ }^{2}=.33\right]$. In order to deconstruct the significant 3-way interaction, separate 2 (occlusion) $\times 2$ (group) ANOVAs for each velocity condition were conducted. No significant main effects or interaction emerged in the slow velocity condition, but a significant interaction was observed in the fast velocity condition, $F(1,16)=6.93, P<.05, \eta_{\mathrm{p}}{ }^{2}=.30$. For the fast velocity condition, a simple main effects analysis indicated that the CTE of the baseball group in the occlusion condition was smaller than it was in the control group $(P<.05)$. Moreover, the control group exhibited significantly larger CTE in the occlusion condition compared with the non-

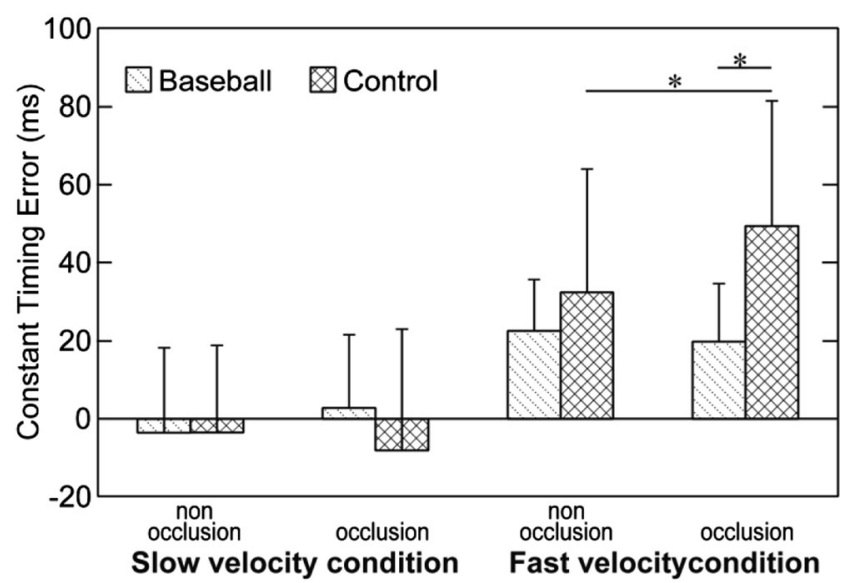

Fig. 4 Mean constant timing error (CTE) in the baseball and control groups within each stimulus condition. Positive CTE values indicate a late response, and negative values indicate an early response. Vertical bars Standard deviation occlusion condition $(P<.01)$. This tendency was not observed in the baseball group.

Next, in this experiment, there was not any random delay between the onset of target motion and the warning stimulus. Therefore, participants in the baseball group might have easily learnt the time interval from onset to arrival of the target motion depending on the target velocity condition. Thus, this might have enabled them to time their response without having to pay attention to the real motion as soon as they knew whether it is fast or slow. In order to test this possibility, we analyzed CTEs in the occlusion condition from the initial trial to the final trial (data only in the occlusion condition were extracted from random trial sequences). CTE was subjected to a two-way repeated measures 2 (velocity: slow vs. fast) $\times 10$ (trial: first trial through final trial) ANOVA. There was no significant main effect of trial $\left[F(9,72)=0.82, P=.60, \eta_{\mathrm{p}}{ }^{2}=\right.$ $.09]$, nor were there any significant interactions regarding the trial factor. According to these results, practice effects did not occur here.

Finally, to investigate whether RM magnitude influenced coincident timing performance, correlation coefficient analysis between RM magnitude and CTE in the occlusion conditions for each group and velocity conditions were performed (Fig. 5). The baseball group exhibited a significant negative correlation between RM magnitude and CTE in the slow $(r=$ $-.83, P<.01)$ and fast velocity conditions $(r=-.66, P<.05)$. The control group exhibited a significant negative correlation between RM magnitude and CTE, but only in the fast velocity condition $(r=-.69, P<.05)$, and not in the slow velocity condition $(r=-.06$, n.s. $)$.

\section{Discussion}

The purpose of the current study was to clarify whether experiences with fast ball sports influence the development of cognitive extrapolation for a moving object. We also sought to clarify the development of cognitive extrapolation as related to coincidental timing performance, especially during interaction with a fast-moving object. Results from the subjective vanishing location (Figs. 2,3) revealed a larger RM effect in the baseball group compared to the control group. These results appear to suggest that experts in fast ball sports can extrapolate the location of a fast-moving object a few hundred milliseconds into the future. Furthermore, RM magnitude and timing errors were strongly negatively correlated in the fast velocity condition in both baseball and control groups, but this negative correlation was observed only in the baseball group in the slow velocity condition (Fig. 5). These negative correlations appear consistent with the idea that cognitive extrapolation is related to controlling response timing as a means of 

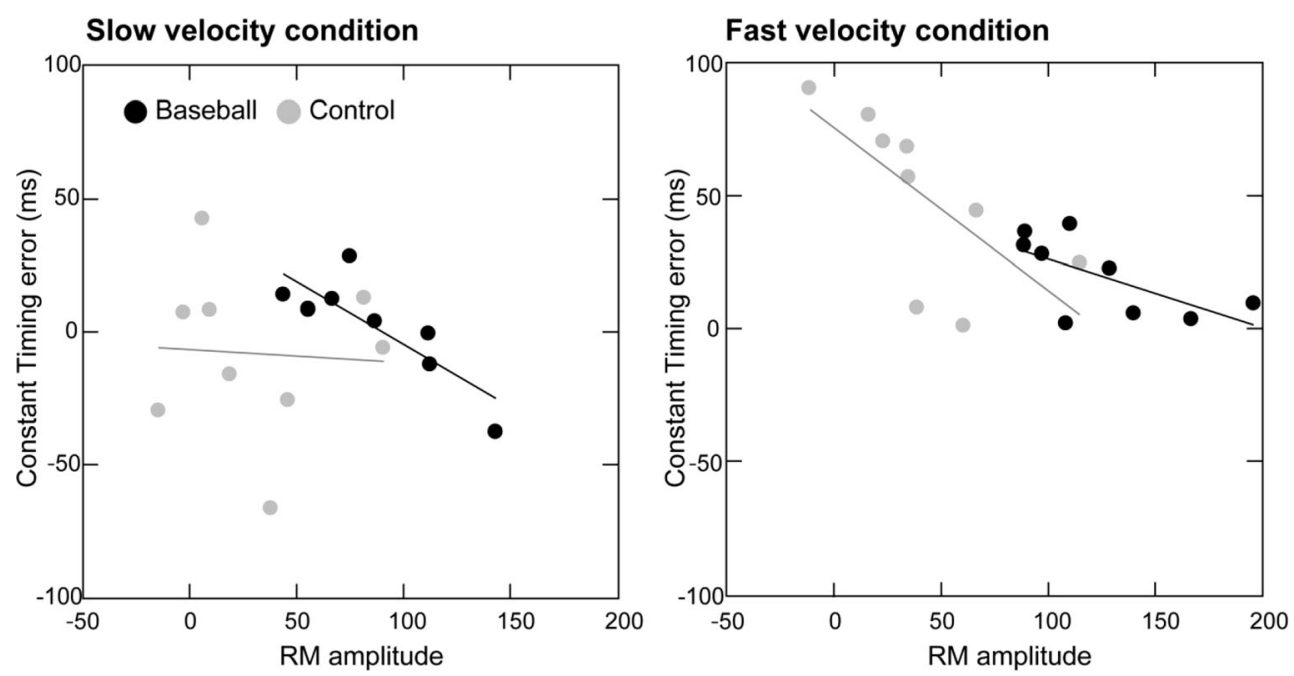

Fig. 5 Relationship between RM magnitude and CTE in each velocity condition

compensating for delays caused by visuo-motor processing (Hubbard, 2005; Nijhawan, 2008).

Previous studies concerning sports expertise effects on RM have reported that experts can perceive the next likely state of a play pattern, while novices cannot (Didierjean \& Marmèche, 2005; Gorman et al., 2011). In addition to this qualitative difference, recent evidence from driving (Blättler et al., 2010, 2012) and basketball activities (Gorman et al., 2012) has shown that experts can perceive states further in the future than can novices (i.e., quantitative difference; see Gilis, Helsen, Catteeuw, \& Wagemans, 2008 for an exception). Consistent with these findings, the present study demonstrated that both baseball and control participants exhibited cognitive extrapolation for a moving target, but the baseball group showed a farther subjective vanishing location than did the control group.

This finding (i.e., that both groups exhibited RM effects) demonstrates that the cognitive extrapolation of a moving target might be a basic primitive function of the visual system. In the present study, moreover, the group differences in RM magnitude were greater in the fast condition than they were in the slow condition (Fig. 3). It has been proposed that the RM effect is modulated by a participant's prior knowledge of an object's typical movement properties stored in long-term memory (Reed \& Vinson, 1996). According to this notion, baseball players in present study might have possessed more refined knowledge regarding the movement properties of a fast-moving target. Therefore, the larger group differences in RM magnitude in the fast velocity condition imply that baseball players may have more developed skills as a function of their experiences in fast ball sports and, consequently, higher resultant knowledge of fast motion properties. On the other hand, the control group may have developed through growth or daily experiences of visual motion perception. Thus, knowledge that contributes to extending farther the subjective vanishing location of a moving target is likely acquired as a function of experiences with increased movement velocity (Akpinar, Devrilmez, \& Kirazci, 2012).

Alternatively, people who do not play fast ball sports may have no need for the more rapid extrapolation required by faster velocities. Thus, they are unpracticed (and thus perhaps unable) to extrapolate at faster velocities. Experience and practice with faster velocities might increase the ability to extrapolate at those faster velocities. Thus, the difference may be not due to learning differences in movement properties per se, but rather to more experience with increased velocities, which subsequently leads to better skill at extrapolating those velocities. Further studies are needed to clarify the properties acquired by the expert to develop cognitive extrapolation.

Oddly, there was a peak at $400 \mathrm{~cm}$ (extreme point) for the baseball group in the fast velocity condition (i.e., $24.1 \%$ of the time). One possible reason for this is that the participants confused the occluded and non-occluded trials fairly regularly. However, the current task presented the stimulus randomly, and the peak at $400 \mathrm{~cm}$ did not occur in the slow condition. Another possibility is that some participants in the baseball group reported seeing the moving target go beyond the end of trackway. That is, in the fast velocity condition, experts may have reported the maximum number (i.e., card number 80 , indicating a location of $400 \mathrm{~cm}$ ) even when they felt the target went beyond the end of trackway. This may explain the high response probability at $400 \mathrm{~cm}$. In any case, this is a very large RM magnitude, even the mean values, compared with RM magnitude in a typical RM experiment. Therefore, we have calculated the visual angle from the occluding location to the subjective vanishing location if participants showed an RM magnitude from $41.6 \mathrm{~cm}$ (average in control group) to $124.1 \mathrm{~cm}$ (average in baseball group) in the fast velocity condition. As a result, the visual angle was from 5.7 to $25.8^{\circ}$. According to these values, if the participants sit directly 
in front of a computer monitor at a distance of $50 \mathrm{~cm}$, which is typically used in RM paradigms, the RM magnitudes would be $5.0-22.9 \mathrm{~cm}$. These values are still larger than previous studies (e.g., Gray \& Thornton, 2001), even in the case of the control group $\left(<1^{\circ}\right)$. This may be because the velocity of moving targets was very high compared with previous studies. In any case, this indicates that baseball players develop cognitive extrapolation for moving targets.

Fast ball sports, such as baseball, are characterized by severe time constraints on intercepting a moving target. If there is no visual function for anticipating or perceiving the future location of a fast-moving ball, neural delays in the visuo-motor system could necessarily cause performance decrements in interceptive timing control. Therefore, if cognitive extrapolation functions to circumvent neural processing delays in timing control, then a larger RM magnitude (i.e., more future extrapolation) enables athletes to circumvent a delay of response errors in interceptive timing control for a fastmoving target (Hubbard, 2005). The present results support this assumption; the significant negative correlation coefficients between RM magnitude and timing errors appeared in the fast velocity condition, which induced a larger positive CTE (i.e., delayed response) in both the baseball and control groups (Fig. 5). That is, cognitive extrapolation becomes more critically functional for timing control when the impact of neural delays is crucial when interacting with the environment (e.g., intercepting a fast-moving ball). Previous studies have reported that TTC underestimations often observed in a slow velocity condition of a PM task (Schiff \& Detwiler, 1979; Schiff \& Oldak, 1990) were effectively eliminated when RM was absent as a function of manipulating the display (Gray \& Thornton, 2001). This evidence also suggests that as time constraints become more severe, the involvement of cognitive extrapolation increases for circumventing neural processing delays in RM tasks with timing control. That is, the introduction of cognitive extrapolation as a compensatory system for timing control would depend on the extent of the induced delay by time constraints.

One of the critical factors for intercepting a moving target is response onset timing (Tresilian, 1995; Tyldesley \& Whiting, 1975). The onset is determined by a visually perceived variable associated with the target. When the perceived variable reaches a criterion value (sometimes called time margin or time threshold relative to the expected interception and/or collision), a motor command is produced (Marinovic, Plooy, \& Tresilian, 2009a, 2009b; Tresilian, 2005; Zago et al., 2009). Marinovic et al. (2009b) reported that movement was prepared in advance and triggered by a visual stimulus event that occurred about $150 \mathrm{~ms}$ before movement onset. In the present study, the elapsed time from the target disappearance to target arrival in the fast condition was $133 \mathrm{~ms}$. In this case, participants should prepare motor processes for an intercepting response before the onset of a response during observation of the target motion (before the target vanishes). As a result, participants are forced to depend on limited information (e.g., extrapolation information) for interceptive timing control.

However, the introduction of a compensatory system for timing control might be a feature of expertise in fast ball sports. In other words, experts rely more heavily on the cognitive extrapolation for executing successful timing control of a motor act compared with novices. Alternatively, experts and novices each rely on extrapolation, but the ability to do so appropriately is better among experts. This is evidenced by the significant within-group correlations between $\mathrm{RM}$ and CTE for both the slow $(r=-.83)$ and fast $(r=-.66)$ conditions in the baseball group, but only in the fast condition in the control group $(r=-.69)$. These results indicate that participants in the control group did not effectively use and/or tune cognitive extrapolation for timing control (Gray \& Thornton, 2001) in the slow condition, and that participants in the baseball group used and/or tuned cognitive extrapolation for timing control irrespective of target velocity.

Moreover, temporal errors in the control group significantly increased in the occlusion condition compared with the non-occlusion condition (Fig. 4). This result may suggest that participants in the control group required at least raw visual information in the coincident timing response, as this increase in temporal errors was not observed in the baseball group. The timing control in the occlusion condition requires sufficient cognitive load to estimate the TTC (DeLucia \& Liddell, 1998; Jagacinski, Johnson, \& Miller, 1983; Tresilian, 1995). The general consensus across numerous studies is that a shorter visible length will yield substantial timing errors, as participants must respond without a continuous view of the moving target, while a longer visible length will yield more accurate responses, as individuals can acquire more detailed information about the target (e.g., Harrold \& Kozar, 2002; Payne, 1986; Whiting, Gill, and Stephenson, 1970). Therefore, larger RM (i.e., longer visible length) after the midpoint of the trajectory (i.e., after the target vanished) would aid timing judgment of the end of the trajectory. That is, our findings suggest that the baseball group may have relied more on and/ or was better tuned to cognitive extrapolations for response timing control than the control group. Taking into account these considerations, RM effects are essentially related to the perception of the future location of a moving object (i.e., TTC estimation), as both groups showed forward extrapolation. On the other hand, information transmitted by cognitive extrapolation working in collaboration with the motor system requires expertise in fast ball sports, which necessitates the interaction of perception and action.

There are two competing theories for understanding the interception of a moving target: the ecological view and the constructivism view (Zago et al., 2009). The former has emphasized that online adjustment during interception is achieved by a continuous coupling between the perceptual 
and motor systems by directly perceiving optical variables such as tau (Bootsma, Houbiers, Whiting, \& van Wieringen, 1991; Bootsma \& van Wieringen, 1990; Le Runigo et al., 2005; Tresilian, 1995) and controlling the required velocity of effector movement (Montagne, Laurent, Durey, \& Bootsma, 1999; Peper, Bootsma, Mestre, \& Bakker, 1994). That is, this theory does not emphasize the use of internal representation of future target motion, but rather, it emphasizes the use of current visual information of target motion to estimate the TTC. In contrast, the latter theory emphasizes that visual cues alone are insufficient for predicting the time and place of interception. Individuals need to be supplemented with prior knowledge or internal representations regarding several features of the dynamic interaction with the moving object (Ikudome, Nakamoto, Yotani, Kanehisa, \& Mori, 2013; Tresilian, 1995; Zago et al., 2009). The present findings suggest that the cognitive extrapolation of a moving target is used and/or tuned by experts in fast ball sports in part for timing control. In contrast, participants in the control group did not effectively use and/or tune cognitive extrapolation for timing control. Rather, they may have partly relied on raw visual information because the $\mathrm{CTE}$ in the occlusion condition increased compared with the non-occlusion condition. Therefore, the availability of cognitive extrapolation and/or raw visual information might depend on the individual's expertise and/or time constraints influencing the estimated neural delay in perceptual and motor processing. Specifically, the present study suggests that several different sources of information and strategies are used and integrated to interpret a moving target, depending on intervening variables (Hubbard, 1997; Tresilian \& Plooy, 2006; Zago \& Lacquaniti, 2005; Zago et al., 2009).

\section{Limitations}

Although the present task was, to some extent, domainspecific to fast ball sports, the movement properties of the moving target in this experiment did not replicate the actual properties of a baseball game (e.g., no deceleration with gravity). Furthermore, baseball players' expertise is likely to be closely attuned to and influenced by factors such as the movement kinematics of the pitcher, the flight path of the ball, and the intention of the batter to actually strike the ball. That is, the present task did not completely capture domain-specific properties of fast ball sports. Although the results showed some degree of an expertise effect, the extent to which this effect was influenced by the lack of specificity within the experimental design is unclear. Further experiments (e.g., comparison between groups under some level of specificity in RM task) are needed to fully understand expertise effects in $\mathrm{RM}$ in fast ball sports.

In order to account for this limitation, an additional control task was conducted using less domain-specificity compared

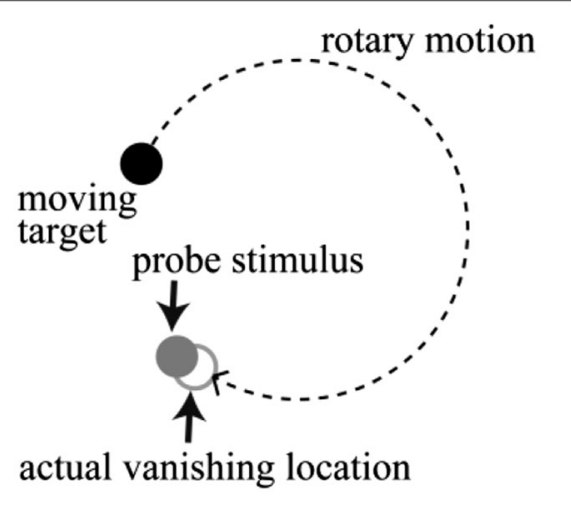

Fig. 6 Schematic representation of experimental stimuli in additional control task. The moving target moved continuously in a circular motion and vanished at a pre-specified location. Participants judged as to whether the moving target vanished at the same location as a presented probe stimulus $(-6,-4,0,+2,+4$, and +6 degrees relative to the vanished location)

with the present task. The task was an RM task with rotating targets (see Fig. 6). The moving target moved continuously in a circular motion and vanished at a pre-specified location. Then, a probe stimulus appeared after $250 \mathrm{~ms}$ at the various locations $\left(-6,-4,0,+2,+4\right.$, and $\left.+6^{\circ}\right)$. Participants ( 11 baseball players, 9 non-baseball players) were required to make a judgment as to whether the moving target vanished at the same location as a presented probe stimulus. Figure 7 shows the probability of a "same" response at each probe stimulus location. The probability was subjected to a 2 (group: baseball or non-baseball) $\times 7$ (probe location: $-6,-4,0,+2,+4,+6$ degrees) mixed measures ANOVA. There was a significant main effect of probe location $\left[F(6,120)=19.89, P<.001, \eta_{\mathrm{p}}{ }^{2}\right.$ $=.50]$, but no significant main effect of group $[F(1,20)=1.25$, $\left.P=.27, \eta_{\mathrm{p}}{ }^{2}=.05\right]$. Further, there was no significant interaction $\left[F(6,120)=0.59, P=.74, \eta_{\mathrm{p}}{ }^{2}=.03\right)$. Post-hoc comparisons showed that the -6 and -4 degree conditions had a smaller probability than the other conditions $(P<.01)$. That is, both groups showed RM effects, but there was no difference between groups. These results indicate that domain-

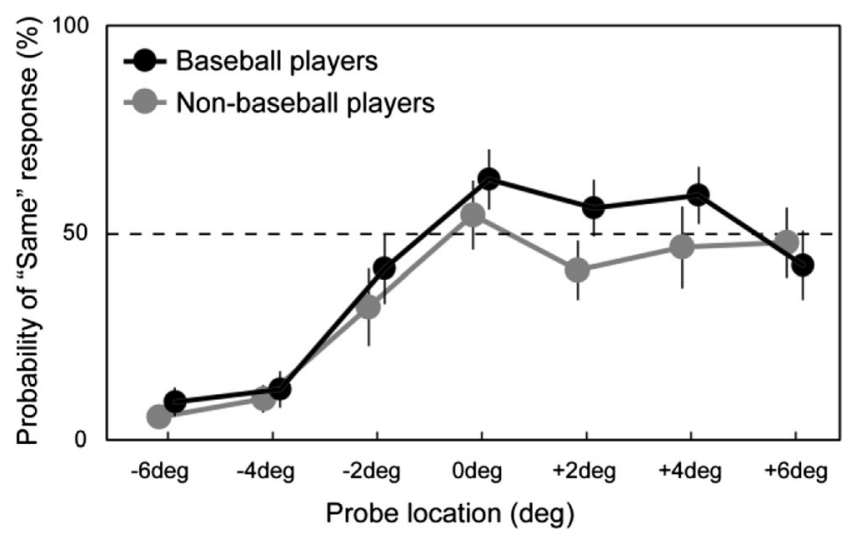

Fig. 7 Probability of "same" response for each probe location. Vertical bars Standard error 
specific experience influences the development of RM magnitude in baseball group.

The difference in acuity that resulted from the vanishing location being visually close or distant might affect RM magnitude. In order to clarify this potential problem, we calculated the visual angle at the occlusion location and at the end of the track. The visual angle of the $5-\mathrm{cm}$ bin oblique to the observer at $200 \mathrm{~cm}$ on the trackway (i.e., occlusion location) is $1.3^{\circ}$ and $2.9^{\circ}$ at $0 \mathrm{~cm}$ on the trackway (i.e., end of the track). The minimum angle of resolution in humans with normal vision such as the present participants is around $0.02^{\circ}$ at a distance of $500 \mathrm{~cm}$ according to a Landolt ring chart. Therefore, if participants were allowed to track the target, the resolution is thought to be sufficient to discriminate the visual angle of the $5-\mathrm{cm}$ bin. On the other hand, if the participants were not allowed to track the target (i.e., participants looked at the target within their peripheral vision), acuity decreased. In our experiment, although we instructed participants to pursue the moving target from the start to the end of the track during the task, we cannot completely rule out the possibility that some participants (and/or some trials) could not track the moving target. The best solution in this case is to record eye movements using an eye-tracking system and/or electrooculogram during the task. Further experiments are necessary to clarify the extent to which this affected the RM magnitudes in this experiment.

\section{Conclusions}

We can conclude that experts in fast ball sports appear to extrapolate the future location of a fast-moving object, and this RM development might relate to coincident timing control. It has been suggested that cognitive extrapolation is required in fast ball sports because of the significant delay in transforming sensory information of a moving target and body motion into a timed motor response (Hubbard, 2005; Nijhawan, 2008; Zago et al., 2009). The present findings further suggest that human visual perception for action depends not only on raw visual information, but also on internal representations that include the expectation for a future object location, perhaps based on prior knowledge (see also, Azevedo Neto \& Teixeira, 2009, 2011; Zago et al., 2009). This cognitive extrapolation of visual perception could compensate for neural delays and physical limitations when following a moving object, anticipating its forthcoming position, and interacting with it appropriately. These findings support evidence from recent behavioral and cognitive neuroscience studies (Blättler et al., 2010, 2011, 2012; Gorman et al., 2011, 2012; Nijhawan, 2008; Nijhawan \& Wu, 2009).
Acknowledgments This work was supported by JSPS KAKENHI Grant Number 26702025 and 25242060.

\section{References}

Akpinar, S., Devrilmez, E., \& Kirazci, S. (2012). Coincidenceanticipation timing requirements are different in racket sports. Perceptual \& Motor Skills, 115, 581-593.

Azevedo Neto, R. M., \& Teixeira, L. A. (2009). Control of interceptive actions is based on expectancy of time to target arrival. Experimental Brain Research, 199, 135-143.

Azevedo Neto, R. M., \& Teixeira, L. A. (2011). Intercepting moving targets: Does memory from practice in a specific condition of target displacement affect movement timing? Experimental Brain Research, 11, 109-117.

Bahill, A. T., \& LaRitz, T. (1984). Why can't batters keep their eyes on the ball? American Scientist, 72, 249-253.

Benguigui, N., Ripoll, H., \& Broderick, M. P. (2003). Time-to-contact estimation of accelerated stimuli is based on first-order information. Journal of Experimental Psychology: Human Perception and Performance, 29, 1083-1101.

Blättler, C., Ferrari, V., Didierjean, A., van Elslande, P., \& Marmèche, E. (2010). Can expertise modulate representational momentum? Visual Cognition, 18, 1253-1273.

Blättler, C., Ferrari, V., Didierjean, A., \& Marmèche, E. (2011). Representational momentum in aviation. Journal of Experimental Psychology: Human Perception and Performance, 37, 1569-1577.

Blättler, C., Ferrari, V., Didierjean, A., \& Marmèche, E. (2012). Role of expertise and action in motion extrapolation from real road scenes. Visual Cognition, 20, 988-1001.

Bootsma, R. J., Houbiers, M. H. J., Whiting, H. T. A., \& van Wieringen, P. C. W. (1991). Acquiring an attacking forehand drive: The effects of static and dynamic environmental conditions. Research Quarterly for Exercise and Sport, 62, 276-284.

Bootsma, R. J., \& van Wieringen, P. C. W. (1990). Timing and attacking forehand drive in table tennis. Journal of Experimental Psychology: Human Learning and Memory, 16, 21-29.

Campbell, F. W., \& Wurtz, R. H. (1978). Saccadic omission: Why we do not see a grey-out during a saccadic eye movement. Vision Research, 18, 1297-1303.

Croft, J. L., Button, C., \& Dicks, M. (2010). Visual strategies of sub-elite cricket batsmen in response to different ball velocities. Human Movement Science, 29, 751-763.

DeLucia, P. R., \& Liddell, G. W. (1998). Cognitive motion extrapolation and cognitive clocking in prediction motion tasks. Journal of Experimental Psychology: Human Perception and Performance, 24, 901-914.

Didierjean, A., \& Marmèche, E. (2005). Anticipatory representation of visual basketball scenes by novice and expert players. Visual Cognition, 12, 265-283.

Freyd, J. J. (1983). The mental representation of movement when static stimuli are viewed. Perception \& Psychophysics, 33, 575-581.

Freyd, J. J., \& Finke, R. A. (1984). Representational momentum. Journal of Experimental Psychology: Learning, Memory, and Cognition, 10, $126-132$.

Freyd, J. J., \& Finke, R. A. (1985). A velocity effect of representational momentum. Bulletin of the Psychonomic Society, 23, 443-446.

Frohlich, C. (1984). Aerodynamics drag crisis and its possible effect on the flight of baseballs. American Journal of Physics, 52, 325-334.

Gilis, B., Helsen, W., Catteeuw, P., \& Wagemans, J. (2008). Offside decisions by expert assistant referees in association football: Perception and recall of spatial positions in complex dynamic events. Journal of Experimental Psychology: Applied, 14, 21-35. 
Gorman, A. D., Abernethy, B., \& Farrow, D. (2011). Investigating the anticipatory nature of pattern perception in sport. Memory \& Cognition, 39, 894-901.

Gorman, A. D., Abernethy, B., \& Farrow, D. (2012). Classical pattern recall tests and the prospective nature of expert performance. Quarterly Journal of Experimental Psychology, 65, 1151-1160.

Gray, R., \& Thornton, I. M. (2001). Exploring the link between time to collision and representational momentum. Perception, 30, $1007-1022$.

Harrold, D., \& Kozar, B. (2002). Velocity, occlusion, and sex of subjects in coincidence of anticipation. Perceptual and Motor Skills, 94, 914-920.

Hubbard, T. L. (1997). Target size and displacement along the axis of implied gravitational attraction: Effects of implied weight and evidence of representational gravity. Journal of Experimental Psychology: Learning, Memory, and Cognition, 23, 1484-1493.

Hubbard, T. L. (2005). Representational momentum and related displacements in spatial memory: A review of the findings. Psychonomic Bulletin \& Review, 12, 822-851.

Hubbard, T. L., \& Bharucha, J. J. (1988). Judged displacement in apparent vertical and horizontal motion. Perception \& Psychophysics, 44, 211-221.

Hubbard, A. W., \& Seng, C. N. (1954). Visual movements of batters. Research Quarterly, 25, 42-57.

Ikudome, S., Nakamoto, H., Yotani, K., Kanehisa, H., \& Mori, S. (2013). Visuo-motor process in movement correction: Role of internal feedback loop. NeuroReport, 24, 879-883.

Jagacinski, R. J., Johnson, W. W., \& Miller, R. A. (1983). Quantifying the cognitive trajectories of extrapolated movements. Journal of Experimental Psychology: Human Perception and Performance, 9, 43-57.

Land, M. F., \& McLeod, P. (2000). From eye movements to actions: How batsmen hit the ball. Nature Neuroscience, 3, 1340-1345.

Le Runigo, C., Benguigui, N., \& Bardy, B. G. (2005). Perception-action coupling and expertise in interceptive actions. Human Movement Science, 24, 429-445.

Lee, S. M. (2010). Does your eye keep on the ball? The strategy of eye movement for volleyball defensive players during spike serve reception. International Journal of Applied Sports Science, 22, 128-137.

Marinovic, W., Plooy, A. M., \& Arnold, D. H. (2012). The influence of visual motion on interceptive actions and perception. Vision Research, 60, 73-78.

Marinovic, W., Plooy, A. M., \& Tresilian, J. R. (2009a). The time course of direction specification in brief interceptive actions. Experimental Psychology, 57, 292-300.

Marinovic, W., Plooy, A. M., \& Tresilian, J. R. (2009b). The utilisation of visual information in the control of rapid interceptive actions. Experimental Psychology, 56, 265-273.

Montagne, G., Laurent, M., Durey, A., \& Bootsma, R. (1999). Movement reversals in ball catching. Experimental Brain Research, 129, 87-92.

Munger, M. P., \& Minchew, J. H. (2002). Parallels between remembering and predicting an object's location. Visual Cognition, 9, 177-194.

Nijhawan, R. (2008). Visual prediction: Psychophysics and neurophysiology of compensation for time delays. Behavioral and Brain Sciences, 31, 179-239.

Nijhawan, R., \& Wu, S. (2009). Compensating time delays with neural predictions: Are predictions sensory or motor? Philosophical Transactions. Series A, Mathematical, Physical, and Engineering Sciences, 367, 1063-1078.

Payne, V. G. (1986). The effects of stimulus runway length on coincidence anticipation timing performance. Journal of Human Movement Studies, 12, 289-295.
Peper, L., Bootsma, R. J., Mestre, D. R., \& Bakker, F. C. (1994). Catching balls: How to get the hand to the right place at the right time. Journal of Experimental Psychology: Human Perception and Performance, 20, 591-612.

Reed, C. L., \& Vinson, N. G. (1996). Conceptual effects on representational momentum. Journal of Experimental Psychology: Human Perception and Performance, 22, 839-850.

Ripoll, H., \& Fleurance, P. (1988). What does keeping one's eye on the ball mean? Ergonomics, 31, 1647-1654.

Ripoll, H., \& Latiri, I. (1997). Effect of expertise on coincident-timing accuracy in a fast ball game. Journal of Sports Science, 15, 573-580.

Schiff, W., \& Detwiler, M. L. (1979). Information used in judging impending collision. Perception, 8, 647-658.

Schiff, W., \& Oldak, R. (1990). Accuracy of judging time to arrival: Effects of modality, trajectory, and gender. Journal of Experimental Psychology: Human Perception and Performance, 16, 303-316.

Selin, C. (1959). An analysis of the aerodynamics of pitched balls. Research Quarterly for Exercise and Sport, 30, 232-240.

Singer, R. N., Williams, A. M., Frehlich, S. G., Janelle, C. M., Radlo, S. J., Barba, D. A., \& Bouchard, L. J. (1998). New frontiers in visual search: An exploratory study in live tennis situations. Research Quarterly for Exercise and Sport, 69, 290-296.

Tenenbaum, G., Sar-El, T., \& Bar-Eli, M. (2000). Anticipation of ball location in low and high-skill performers: A developmental perspective. Psychology of Sport and Exercise, 1, 117-128.

Tresilian, J. R. (1995). Perceptual and cognitive processes in time-tocontact estimation: Analysis of prediction-motion and relative judgment tasks. Perception \& Psychophysics, 57, 231-245.

Tresilian, J. R. (2005). Hitting moving targets: Perception and action in the timing of fast interceptions. Perception \& Psychophysics, 67, 129-149.

Tresilian, J. R., \& Plooy, A. (2006). Systematic changes in the duration and precision of interception in response to variations in movement amplitude and effector size. Experimental Brain Research, 171, 421-435.

Tyldesley, D. A., \& Whiting, H. T. A. (1975). Operational timing. Journal of Human Movement Studies, 1, 172-177.

Verfaillie, K., \& d'Ydewalle, G. (1991). Representational momentum and event course anticipation in the perception of implied periodical motions. Journal of Experimental Psychology: Learning, Memory, and Cognition, 17, 302-313.

Volkmann, F. C., Schick, A. M., \& Riggs, L. A. (1968). Time course of visual inhibition during voluntary saccades. Journal of the Optical Society of America, 58, 562-569.

Whiting, H. T. A., Gill, E. B., \& Stephenson, J. M. (1970). Critical time intervals for taking flight information in ball-catching task. Ergonomics, 13, 265-272.

Williams, A. M., Davids, K., \& Williams, J. G. (1999). Visual perception and action in sport. London: $\mathrm{E} \& \mathrm{FN}$ Spon.

Williams, A. M., \& Ericsson, K. A. (2005). Perceptual-cognitive expertise in sport: Some considerations when applying the expert performance approach. Human Movement Science, 24, 283-307.

Williams, T., \& Underwood, J. (1986). The science of hitting. New York: Simon and Schuster.

Zago, M., \& Lacquaniti, F. (2005). Cognitive, perceptual and actionoriented representations of falling objects. Neuropsychologia, 43, $178-188$.

Zago, M., McIntyre, J., Senot, P., \& Lacquaniti, F. (2009). Visuo-motor coordination and internal models for object interception. Experimental Brain Research, 192, 571-604. 